\title{
Afrikanischer Menschenrechtsschutz - Neuere Entwicklungen und Perspektiven
}

\author{
Der Afrikanische Gerichtshof der Rechte des Menschen und der Völker \\ und die Mauritius-Erklärung der Organisation der Afrikanischen Einheit ${ }^{1}$
}

Von Michaela Wittinger, Saarbrücken

Die neueren Entwicklungen im afrikanischen Menschenrechtsschutz sind durch zwei entscheidende Ereignisse geprägt: erstens durch die Schaffung eines Afrikanischen Menschenrechtsgerichtshofes ${ }^{2}$ und zweitens durch die Menschenrechtserklärung und den Handlungsplan $^{3}$ der Organisation der afrikanischen Einheit (OAU). Der vorliegende Beitrag untersucht die Frage, welche Perspektiven sich hieraus für den afrikanischen Menschenrechtsschutz ergeben. Insbesondere ist dabei zu berücksichtigen, welche Folgen sich für die Afrikanische Charta der Rechte des Menschen und der Völker ${ }^{4}-$ das entscheidende afrikanische Menschenrechtsinstrument - ergeben und inwiefern diese fortentwickelt wird. Zunächst werden die Charakteristika des neuen Afrikanischen Menschenrechtsgerichtshofes vorgestellt.

Der Beitrag beruht auf dem von der Verfasserin auf der Jahrestagung des Arbeitskreises für Überseeische Verfassungsvergleichung (vom 22.-24. Juni in Heidelberg) gehaltenen Vortrag. Der Vortragsstil wurde weitgehend beibehalten.

Protocol to the African Charter on Human and Peoples' Rights on the Establishment of an African Court on Human and Peoples' Rights v. 9.6.1998, abgedruckt z.B. in Human Rights Law Journal 20 (1999), 727 ff.; im Folgenden: Protokoll zur Gründung des Afrikanischen Menschenrechtsgerichtshofes.

Declaration and Plan of Action, CONF/HRA/DECL (I), abgedruckt in Revue Africaine du Droit International Comparé 11 (1999), 352 ff.; im Internet abrufbar unter http://www.ncb.intnet.mu/ $\mathrm{mfa} / \mathrm{oau} / \mathrm{decpl}$.htm. Zur Mauritius-Erklärung und zur Praxis der Afrikanischen Menschenrechtskommission Naldi, Zeitschrift für ausländisches öffentliches Recht und Völkerrecht 60 (2000), $715 \mathrm{ff}$.

4 Vom 27.6.1981, in Kraft seit 21.10.1986, OAU/Doc.CAB/LEG/67/3/Rev.5., frz. Fassung in Revue Universelle des Droits de l'Homme 1 (1989), 484 ff.; engl. Fassung z.B. in International Legal Materials 21 (1982), 59 ff.; deutsche Übersetzung z.B. in Europäische Grundrechte Zeitschrift 13 (1986), 677 ff.; im Folgenden: AfrC. 


\section{Der Afrikanische Menschenrechtsgerichtshof}

\section{Hintergrund}

Der Vorschlag, einen unabhängigen Afrikanischen Menschenrechtsgerichtshof zu schaffen, stammt bereits aus dem Jahre 1961 und wurde im Zuge der Entstehung ${ }^{5}$ der Afrikanischen Menschenrechtscharta immer wieder thematisiert. Die Befürworter eines Gerichtshofes konnten sich aber damals nicht durchsetzen, und man einigte sich lediglich auf ein nicht judikatives Organ: die Afrikanische Menschenrechtskommission. Diese hat drei Aufgaben: einmal die Förderung der Menschenrechte durch Information der Öffentlichkeit über die Charta, zum zweiten die Prüfung der Staatenberichte, in denen die Mitgliedstaaten im Zwei-Jahres-Rhythmus Rechenschaft über die nationale Menschenrechtslage geben und zum dritten und nicht zuletzt die Prüfung von Beschwerden über Menschenrechtsverletzungen, welche von Individuen oder von Nichtregierungsorganisationen (NGOs) erhoben werden können. ${ }^{6}$ Nach Prüfung der Zulässigkeit einer Individualbeschwerde kann sich die Afrikanische Menschenrechtskommission allerdings nur dann weiter mit der Beschwerde befassen, wenn sie von der Versammlung der Staats- und Regierungschefs der OAU hierzu aufgefordert wird. Ferner steht der Kommission als einzige Sanktionsmöglichkeit im Falle einer Verletzung der Afrikanischen Menschenrechtscharta die Bekanntgabe der Kommissionsentscheidung als Anhang zu ihrem Jahresbericht zur Verfügung, der der OAU-Versammlung vorgelegt wird. Auch eine Veröffentlichung der Aktivitätsberichte ${ }^{7}$ der Kommission ist von der Zustimmung dieses politischen Gremiums abhängig. Damit ist das Beschwerdeverfahren, trotz der Auflockerungen in der Verfahrensordnung ${ }^{8}$ noch immer von dem Prinzip der Vertraulichkeit beherrscht, das nur mit Zustimmung der OAU-Versammlung der Staats- und Regierungschefs aufgehoben werden kann.

Für die damalige Ablehnung eines Afrikanischen Menschenrechtsgerichtshofs gab es zwei Gründe. ${ }^{9}$ Den offiziellen, dass eine Kommission eher zur Vermittlung und zum Ausgleich geeignet sei, entsprechend der afrikanischen Rechtstradition einen Streit durch Diskussion

Hierzu z.B.: Kunig, German Yearbook of International Law 25 (1982), 143 ff.; Kodjo, Human Rights Law Journal 11 (1990), 272 ff.

Siehe Art. 45, 58 und 62 AfrC; zur Kommission und zu ihren Verfahren insgesamt Ankumah, The African Commission on Human and Peoples' Rights, Practice and Procedures, The Hague et al. 1996.

Zur Entwicklung in den Aktivitätsberichten Umozurike, The African Charter on Human and Peoples' Rights, The Hague et al. 1997, S. 79.

Siehe Art. 32 Amended Rules of Procedure angenommen am 6.10.1995, abgedruckt in Human Rights Law Journal 18 (1997), 154 ff.

9

Hierzu: M'Baye, Les Droits de l'Homme en Afrique, Paris 1992, S. 164; Ouguergouz, La charte africaine des droits de l'homme et des peuples, Genève/Paris 1993, S. 386 ff. 
anstatt durch kontradiktorisches Urteil beizulegen, und den inoffiziellen, vielleicht wahren Grund, dass sich die jungen unabhängigen afrikanischen Staaten keinem übergeordneten Gericht unterwerfen wollten, da dies wieder die Einbuße eines Teils ihrer Souveränität bedeutet hätte. Erst Anfang der 90er Jahre kam es zu konkreten erneuten Versuchen, eine Judikative zu schaffen - auch unter dem Eindruck der genannten Schwächen der Afrikanischen Kommission: der mangelnden Publizität, fehlender Sanktionsmöglichkeiten und der Abhängigkeit von der OAU-Versammlung der Staats- und Regierungschefs.

Im Juni 1998 wurde das Protokoll zur Afrikanischen Charta über die Gründung des Menschenrechtsgerichtshofes ${ }^{10}$ durch die OAU angenommen. In Kraft tritt das Protokoll nach der 15. Ratifikation. ${ }^{11}$ Bisher haben es 30 der 51 Mitgliedstaaten der Afrikanischen Menschenrechtscharta unterzeichnet und drei Staaten ratifiziert. ${ }^{12}$ Bevor auf die Kompetenzen des Afrikanischen Menschenrechtsgerichtshofs eingegangen wird, ist zunächst ein kurzer Blick auf seine Zusammensetzung ${ }^{13}$ zu werfen.

\section{Aufbau}

Der Gerichtshof wird aus elf Richtern bestehen, anders als z.B. beim Europäischen Gerichtshof für Menschenrechte wird also nicht jeder Staat einen Richter stellen. ${ }^{14}$ Ferner ist der nationale Richter in Fällen gegen seinen eigenen Staat vom Verfahren ausgeschlossen. Man hat hier offenbar auf Probleme der Unparteilichkeit, die bei der Afrikanischen Menschenrechtskommission aufgetreten sind, reagiert, deren Mitglieder mitunter Regierungsmitglieder der jeweiligen Staaten sind. ${ }^{15}$ Die in diesem Zusammenhang ebenfalls angestrebte Einführung einer Vollbeschäftigung der Richter konnte aufgrund der begrenzten Finanzmittel - ein Problem, auf das immer wieder zurückzukommen sein wird - nicht verwirklicht werden; ein weiterer Grund mag auch in der nicht allzu großen Anzahl der zu erwartenden Fälle liegen - so hatte die Kommission in ihren ersten zehn Tätigkeitsjahren (von 1987 bis 1997) circa 200 Individualbeschwerden zu bewältigen. ${ }^{16}$ Es ist aber vorgese-

Zum Nachweis Fn. 2.

11

12

13 
hen, dass die Teilzeitvariante später von der OAU-Versammlung der Staats- und Regierungschefs aufgehoben werden kann. ${ }^{17}$

\section{Kompetenzen}

Der Afrikanische Menschenrechtsgerichtshof hat nach dem Protokoll zwei Funktionen: zum einen kann er Gutachten auf Antrag eines Staates erstatten ${ }^{18}$, zum anderen ist er für Beschwerden zuständig: für Staatenbeschwerden ${ }^{19}$ und Individualbeschwerden ${ }^{20}$. Individualbeschwerden - in der Praxis sind nur sie entscheidend, das zeigen das europäische und das inter-amerikanische System - können nach dem Protokoll auf zwei Wegen vor den Gerichtshof gebracht werden:

Erstens - und das ist der Regelfall - kann er in Beschwerden angerufen werden, die zunächst von der Afrikanischen Menschenrechtskommission geprüft wurden, entsprechend dem früheren europäischen Verfahren und dem geltenden inter-amerikanischen System ${ }^{21}$ : also nur die Kommission und Staaten, die im Kommissionsverfahren betroffen sind, nicht aber das Individuum oder eine NGO sind berechtigt, den Gerichtshof anzurufen. ${ }^{22}$

Zweitens ist auch eine direkte Klage vor dem Gerichtshof durch den Einzelnen oder eine NGO möglich. Da die actio popularis wie vor der Afrikanischen Menschenrechtskommission zulässig ist ${ }^{23}$, wird hier, wie sich schon in den Verfahren vor der Kommission gezeigt hat $^{24}$, für internationale und afrikanische NGOs ein wichtiges Betätigungsfeld bestehen, da der Einzelne nicht selten Repressionen befürchtet oder nicht über die Information oder

Art. 15 Abs. 4 des Protokolls zur Gründung des Afrikanischen Menschenrechtsgerichtshofes.

Art. 4 des Protokolls zur Gründung des Afrikanischen Menschenrechtsgerichtshofes.

Art. 5 Abs. 1 b), c) und d) des Protokolls zur Gründung des Afrikanischen Menschenrechtsgerichtshofes.

Art. 5 Abs. 3 des Protokolls zur Gründung des Afrikanischen Menschenrechtsgerichtshofes.

Zum Vergleich der drei regionalen Schutzsysteme Wittinger, Juristische Ausbildung 21 (1999), $405 \mathrm{ff}$.

Art. 5 Abs. 1 und 3 des Protokolls zur Gründung des Afrikanischen Menschenrechtsgerichtshofes.

Art. 6 Abs. 2 Protocol to the African Charter on Human and Peoples' Rights on the Establishment of an African Court on Human and Peoples' Rights i.V.m. Art. 56 AfrC.

24

Siehe z.B. die von NGOs eingelegte Beschwerde 60/90, Constitutional Rights Project v. Zaire (on behalf of Wahab Akuma, G. Adeaga and others), Opinion of 3 November 1994, abgedruckt in Human Rights Law Journal 18 (1997), 28 f;; weitere Beispiele ebenda, 29 ff.; zum Verhältnis NGOs - Afrikanische Menschenrechtskommission auch Murray, ebenda, 17. 
Mittel $^{25}$ verfügt, eine Beschwerde zu betreiben. Entscheidend bei dieser direkten Klagemöglichkeit durch den Einzelnen oder eine NGO ist, dass sie nur möglich ist, wenn der Vertragsstaat, gegen den sich die Beschwerde richtet, in einer speziellen Erklärung die Zuständigkeit des Gerichtshofs für diese Fälle anerkannt hat ${ }^{26}$ : das Protokoll bringt damit kein automatisches Individualklagerecht mit sich. Ein Staat kann also das Protokoll unterzeichnen und ratifizieren, ohne diese spezielle Erklärung abzugeben; für das Individuum gibt es in diesem Falle keinen Weg, die Befassung des Gerichtshofs mit seiner Beschwerde zu erzwingen. Die Situation entspricht also der ursprünglichen Vorgabe der Europäischen Menschenrechtskonvention (EMRK), noch vor Inkrafttreten des 9. Zusatzprotokolls zur EMRK. $^{27}$ Mit der Situation im europäischen System seit der Geltung des Reformprotokolls Nr. 11 ist sie damit nicht vergleichbar ${ }^{28}$. Diese zurückhaltende Lösung und zusätzliche Hürde einer speziellen Unterwerfungserklärung sollte auch zögernden afrikanischen Staaten die Annahme des Protokolls erleichtern, um den Preis, dass es lange dauern kann, bis ein direktes Anrufungsrecht für das Individuum vor dem Afrikanischen Menschenrechtsgerichtshof erreicht sein wird.

Frühere Protokollfassungen ${ }^{29}$ zur Gründung des Afrikanischen Menschenrechtsgerichtshofs hatten andere Modelle für das Individualverfahren vorgesehen. Insbesondere NGOs engagierten sich für die Verankerung eines direkten Anrufungsrechtes zugunsten des Individuums. So wurde vorgeschlagen, dass der Einzelne eine Beschwerde vor dem Gerichthof erheben kann, wenn „,besondere Gründe“ vorliegen. Dies hätte dem Gerichtshof die flexible Möglichkeit eröffnet, diese „,besonderen Gründe“ sehr weit auszulegen - ähnlich, wie es die Afrikanische Menschenrechtskommission ihrerseits im Beschwerdeverfahren bei der Vorlage ihrer Entscheidungen an die OAU-Versammlung handhabt. Denn nach dem Wortlaut der Afrikanischen Menschenrechtscharta betrifft die Vorlage über ,andere als Staatenbeschwerden“ nur solche über ,besondere Fälle, die auf eine Häufung von massiven oder schwerwiegenden Menschenrechtsverletzungen hindeuten“. ${ }^{30}$ In der Praxis betrachtet die Kommission aber Menschenrechtsverletzungen regelmäßig als schwerwiegend in diesem Sinne. Möglicherweise befürchteten die Staaten eine vergleichbar extensive Ausschöpfung seitens des Afrikanischen Menschenrechtsgerichtshofs, die unterbunden werden sollte.

Zur Möglichkeit der Prozesskostenhilfe vor dem Afrikanischen Menschenrechtsgerichtshof siehe Art. 10 Abs. 2 des Protokolls zur Gründung des Afrikanischen Menschenrechtsgerichtshofes. Art. 34 Abs. 6 des Protokolls zur Gründung des Afrikanischen Menschenrechtsgerichtshofes. V. 6.11.1990, in Kraft seit 1.10.1994.

V. 11.5.1994, in Kraft seit 1.11.1998; zur Reform nur Schlette, Zeitschrift für ausländisches öffentliches Recht und Völkerrecht 56 (1996), 914 ff. 
Insgesamt ist zum Beschwerdeverfahren festzuhalten, dass die Sitzungen des Gerichtshofs nach dem Protokoll regelmäßig öffentlich sind. ${ }^{31}$

So restriktiv einerseits bei der Individualbeschwerde verfahren wurde, so großzügiger war man in Bezug auf den Umfang des Rechts, das der Gerichtshof bei der Beurteilung der Beschwerden anwenden kann. Der Gerichtshof kann die Beschwerden nicht nur am Maßstab der Afrikanischen Menschenrechtscharta überprüfen, sondern anhand ,jedes anderen relevanten Menschenrechtsinstruments“, das der betroffene Staat ratifiziert hat. ${ }^{32}$ Dies bietet den Vorteil, dass der Gerichtshof auch Rechte heranziehen kann, die in der Afrikanischen Menschenrechtscharta selbst nicht enthalten sind, wie z.B. Eheschutzvorschriften. Auf der anderen Seite besteht die Schwierigkeit, dass Divergenzen zur Spruchpraxis anderer internationaler Organe auftreten können, die originär zur Interpretation des entsprechenden Menschenrechtsdokuments berufen sind, wie z.B. zum UN-Menschenrechtsausschuss.

Stellt der Gerichtshof eine Menschenrechtsverletzung fest, soll das Urteil auch die Maßgabe enthalten, wie die Situation zu beseitigen ist ${ }^{33}$ - anders als der Europäische Menschenrechtsgerichtshof kann er also genaue Vorgaben machen, z.B. dass ein Strafverfahren neu durchzuführen ist. Es ist damit nicht dem Staat überlassen, wie er das Urteil umsetzt. ${ }^{34}$ Daneben kann der Afrikanische Gerichtshof - wie der Europäische Menschenrechtsgerichtshof - eine seitens des Staates zu leistende Entschädigungszahlung bestimmen. ${ }^{35}$

Wie sieht es nun mit der Durchsetzung der Urteile aus? Die Überprüfung, ob die Urteile befolgt wurden, obliegt dem Gerichtshof selbst ${ }^{36}$, während in Straßburg das Ministerkomitee die Durchsetzung kontrolliert. ${ }^{37}$ Als Sanktion kann der Afrikanische Menschenrechtsgerichtshof allerdings nur die Staaten in seinem Aktivitätsbericht benennen, die seine Urteile nicht umgesetzt haben; seinen Bericht legt er der OAU-Versammlung vor. Entscheidend ist, dass dieser Bericht öffentlich ist - etwas Gegenteiliges steht jedenfalls nicht im Protokoll, so dass als Sanktionsmittel im Ergebnis die Publizität zur Verfügung steht: nicht mehr, etwa der Ausschluss aus der OAU, wie er für den Europarat - allerdings durch das politische Organ des Ministerkomitees - erfolgen kann ${ }^{38}$, aber auch nicht weniger, wie es

Art. 10 Abs. 1 des Protokolls zur Gründung des Afrikanischen Menschenrechtsgerichtshofes.

Vgl. Art. 3 des Protokolls zur Gründung des Afrikanischen Menschenrechtsgerichtshofes.

Art. 21 des Protokolls zur Gründung des Afrikanischen Menschenrechtsgerichtshofes.

Vgl. Art. 46 EMRK, hierzu Wittinger, Neue Juristische Wochenschrift 53 (2000), 1238 f. m.w.N. Vgl. Art. 41 EMRK.

Art. 31 des Protokolls zur Gründung des Afrikanischen Menschenrechtsgerichtshofes.

Vgl. Art. 46 Abs. 2 EMRK.

Art. 8 Europaratssatzung v. 5.5.1949. 
bei der Afrikanischen Menschenrechtskommission und ihrer mangelnden Selbstbestimmung bezüglich der Publizität ihrer Entscheidungen der Fall ist.

\section{Bewertung}

Bei der Bewertung des Afrikanischen Menschenrechtsgerichtshofes ist damit festzuhalten, dass die Schwierigkeiten, mit denen das bisherige Schutzsystem der Afrikanischen Menschenrechtscharta behaftet war, mit dem Protokoll behoben wurden - durch Schaffung von Öffentlichkeit und durch Einführung einer eigenständigen Kontrolle der Umsetzung der Entscheidungen, ohne eine Abhängigkeit von OAU-Organen. Andererseits wurde der Schutz des Individuums nur halbherzig verwirklicht, indem kein automatisches Anrufungsrecht eingeführt wurde. Nicht außer Acht darf dabei allerdings bleiben, dass es sich im Menschenrechtsschutz insgesamt um langwierige Prozesse handelt. Auch im Rahmen der EMRK wurden verschiede Entwicklungsstufen durchgemacht, und hat es fast 50 Jahre gedauert, bis mit dem Reformprotokoll Nr. 11 ein direktes, alle Mitgliedstaaten bindendes Klagerecht für das Individuum geschaffen wurde. Die Maßstäbe an die Entwicklungen im afrikanischen Menschenrechtsschutz dürfen daher nicht überzogen werden. Ferner ist zu bedenken, dass es nicht alleine der Gerichtshof sein wird, der zur Verbesserung der Menschenrechtslage in den afrikanischen Staaten beitragen kann. Dem Einwand, unter dem Eindruck der Änderungen im europäischen System und der Abschaffung der Europäischen Menschenrechtskommission, auch im afrikanischen Menschenrechtssystem für die Existenz nur einer einzigen Menschenrechtsinstanz zu votieren und die Afrikanische Menschenrechtskommission aufzulösen, ist damit eine Absage zu erteilen. ${ }^{39}$ Dies ist in den Gegebenheiten auf dem afrikanischen Kontinent begründet. Denn der Individualschutz gerade durch ein internationales Gericht, der in Europa als der Erstrebenswerteste erscheinen mag, ist auch nur dann so wertvoll, wenn nationale Instanzen zur Verfügung stehen, die die internationalen Urteile umsetzen und dem Bürger Rechtsschutz gewähren. Unter militärischen Diktaturen und in nur schwachen demokratischen Strukturen dagegen stellt sich die Situation anders dar. Dies gilt ebenso für Staaten, die von ethnischen Kriegen und Bürgerkriegssituationen erschüttert werden. In solchen Staaten ist gerade auch die Arbeit der Afrikanischen Menschenrechtskommission von Bedeutung, die über den individuellen Fall hinaus, Missstände aufzeigen und die Lage der Menschenrechte in einem Land insgesamt untersuchen kann und so Reaktionen seitens der Mitgliedstaaten zur Verbesserung erreichen kann. Als Organ, das kein Gericht ist und kein Urteil abfassen muss, verfügt die Kommission auch eher über Spielräume im informellen Bereich, die genutzt werden können.

Allerdings ist angesichts der gravierenden Finanzierungsschwierigkeiten der Afrikanischen Menschenrechtskommission durchaus die Frage gerechtfertigt, wie in der Praxis nun zwei 
Organe zum Schutze der Menschenrechte funktionsfähig gehalten werden können. Damit ist abschließend die faktische Seite zu berücksichtigen: die schwierige Finanzlage, der sich der Afrikanische Menschenrechtsgerichtshof gegenüber sehen wird. Finanziert werden soll er durch die $\mathrm{OAU}^{40}$, also nicht direkt von den Unterzeichnerstaaten; auch dies sollte die Zustimmung der Mitgliedstaaten zum Protokoll erleichtern. Vergegenwärtigt man sich die Situation bei der Afrikanischen Menschenrechtskommission und die dortige unzureichende personelle und technische Ausstattung, muss - realistisch betrachtet - die Zukunft des Gerichtshofs eher skeptisch beurteilt werden. Für die Afrikanische Menschenrechtskommission, die ebenfalls direkt durch die OAU finanziert wird, hat der UN High Commissioner for Human Rights festgestellt, dass die geringen finanziellen Ressourcen der Kommission das Haupthindernis für die Verwirklichung ihrer Arbeit darstellen. ${ }^{41}$ Es bleibt zu befürchten, dass sich die Lage des Afrikanischen Menschenrechtsgerichtshofs ähnlich prekär gestalten wird.

\section{Die Menschenrechtserklärung und der Handlungsplan der Ersten OAU- Ministerkonferenz über Menschenrechte in Grand Bay (Mauritius), angenommen am 16. April 1999 auf der Ersten Ministerkonferenz für Menschenrechte in Grand Bay (Mauritius) ${ }^{42}$}

\section{Hintergrund: die Instabilität auf dem afrikanischen Kontinent}

Die ausführliche Präambel der Mauritius-Erklärung gibt Aufschluss über den Hintergrund des Dokuments. Es ist die Erkenntnis, dass der Schutz der Menschenrechte die Basis für die weitere Entwicklung des Afrikanischen Kontinents darstellt. Angesichts der Erfahrungen ethnischer Konflikte, des Genozids, von Instabilität, Naturkatastrophen und Wirtschaftskrisen werden die Stärkung und der Schutz der Menschenrechte als Schlüssel für Frieden, für die Beseitigung von Konflikten, für Demokratisierung und Rechtsstaatlichkeit angesehen.

\section{Inhalt der Mauritius-Erklärung und des Aktionsplanes: Klarheit auch für die} Afrikanische Charta der Rechte des Menschen und der Völker?

Die Mauritius-Erklärung kann nicht losgelöst betrachtet werden. Sie fügt sich in das bestehende Geflecht afrikanischer Menschenrechtsdokumente ein und ist insbesondere im Zusammenhang mit der Afrikanischen Charta der Rechte des Menschen und der Völker zu

\footnotetext{
40

Art. 32 des Protokolls zur Gründung des Afrikanischen Menschenrechtsgerichtshofes.

41 UN Doc. E/CN.4/1999/93, § 6; hierzu auch Krisch, Zeitschrift für ausländisches öffentliches Recht und Völkerrecht 58 (1998), 734, Anm. 152.

42

Zum Nachweis Fn. 3; im Folgenden: Mauritius-Erklärung.
} 
betrachten, auf die in der Erklärung immer wieder verwiesen wird. ${ }^{43}$ Zwei Charakteristika der Mauritius-Erklärung sind insoweit vorab festzuhalten, auf welche im Folgenden näher einzugehen ist: Zum einen werden die Wertentscheidungen der Afrikanischen Menschenrechtscharta zur Bedeutung der wirtschaftlichen Rechte beibehalten, zum anderen bringt die Mauritius-Erklärung Klarheit für die Auslegungsschwierigkeiten in der Afrikanischen Charta im Bereich der ,afrikanischen Traditionen“ und für die Rechte von Frauen und Kindern.

Im Einzelnen besteht die Mauritius-Erklärung aus 29 Regelungspunkten. Drei inhaltliche Schwerpunkte lassen sich dabei herausarbeiten: Erstens wird in der Erklärung insgesamt die Bedeutung der Menschenrechte für die afrikanischen Staaten betont und die Gründe für die Menschenrechtsverletzungen in Afrika aufgelistet, zweitens unterstreicht die Erklärung immer wieder die Bedeutung der Afrikanischen Menschenrechtscharta und schlägt Maßnahmen zur Stärkung der Afrikanischen Menschenrechtskommission vor, drittens enthält die Erklärung auch einen Aktionsplan und wendet sich insofern an die Staaten, die OAU und die internationale Gemeinschaft, die jeweils Maßnahmen zur Verbesserung der Menschenrechtslage in Afrika ergreifen sollen.

\section{a) Die Bedeutung der Menschenrechte für die afrikanischen Staaten: Universalität und afrikanische Traditionen}

Die Mauritius-Erklärung beginnt mit der Aufforderung an die Staaten, die wirtschaftlichen, sozialen und kulturellen Rechte gleichermaßen wie die zivilen und politischen Rechte zu verwirklichen. ${ }^{44}$ Wie schon in der Präambel ${ }^{45}$ wird wieder das Recht auf Entwicklung unterstrichen, ferner das Recht auf eine gesunde Umwelt und auf Frieden. ${ }^{46}$ Mit der Betonung der Bedeutung der wirtschaftlichen Rechte und der Rechte der sog. dritten Generation wird damit die Tradition der Afrikanischen Charta fortgesetzt, sich nicht vorrangig an den klassischen Individualabwehrrechten europäischer oder internationaler Menschenrechtskataloge zu orientieren, sondern mittels des Schutzes wirtschaftlicher Rechte und des Rechts auf Entwicklung, die Anhebung des Entwicklungsstandes der afrikanischen Staaten zu erreichen und ebenso nicht nur den Einzelnen mit Rechten auszustatten, sondern Rechte der Völker zu verbriefen. ${ }^{47}$ 
Es fällt auf, dass die Mauritius-Erklärung zum Verhältnis Universalität einerseits und afrikanische Traditionen und Wertvorstellungen andererseits klarer Stellung bezieht als die Afrikanische Menschenrechtscharta. Bereits zu Beginn enthält die Mauritius-Erklärung das Bekenntnis, dass die Menschenrechte universell und unteilbar sind ${ }^{48}$ - eine Aussage, die sich in der Afrikanischen Menschenrechtscharta nicht findet. Die Afrikanische Charta ist noch sehr deutlich von der Ambivalenz geprägt, afrikanische Eigenständigkeit und afrikanisches Rechtsverständnis zu wahren und zugleich nicht abseits des internationalen Menschenrechtsschutzes zu stehen, sondern sich darin zu integrieren ${ }^{49}$; dies zeigt sich daran, dass in der Charta zum einen weitreichende Verweise auf internationale Menschenrechtsdokumente enthalten $\operatorname{sind}^{50}$, die bei stringenter Auslegung dazu führen, dass internationale Vorschriften von der Afrikanischen Menschenrechtskommission bei der Beurteilung von Beschwerden direkt angewandt werden können ${ }^{51}$, auf der anderen Seite betont die Charta, dass ,afrikanische Traditionen“ bewahrt werden müssen und verwendet immer wieder die Begriffe „Tradition und afrikanische traditionelle Werte ${ }^{\text {“62 }}$, die es z.B. seitens der Familie zu schützen gelte, ohne dass feststeht, was hierunter zu verstehen ist. ${ }^{53}$ Angesichts aber der Existenz vieler Traditionen, die zu Lasten von Frauen und Mädchen gehen und die im Gewohnheitsrecht rechtlich zementiert sind, z.B. im Kontext mit der Ehe - Kinderehen, Verheiratung ohne Einverständnis, Brautpreis, gewohnheitsrechtlicher Ausschluss von Frauen, Land erben und erwerben zu können, rechtlose Stellung als Witwe oder anderer Bräuche - wie z.B. die Geschlechtsverstümmelung, stellt sich in der Charta immer wieder die Frage, ob auch solche Traditionen mit ihr vereinbar sein können. ${ }^{54}$ In der Mauritiusdeklaration hingegen wird ausdrücklich bekräftigt, dass es einen Kanon von Werten gibt, den alle Kulturen teilen: ,die Heiligkeit des Lebens und die Menschenwürde, Toleranz, der Wunsch nach Freiheit, Ordnung, Gerechtigkeit, Wachstum und Stabilität" "55 und dass in diesem Sinne positive traditionelle und kulturelle afrikanische Werte in den Menschenrechtsschutz aufgenommen werden müssten, um sie an die künftigen Generationen weiterzugeben. In der Mauritius-Erklärung sprechen sich die OAU-Minister folglich nur zugunsten von positiven Traditionen aus, die mit den vorgenannten universellen Werten im Ein-

Punkt 1 der Mauritius-Erklärung.

Zur „Afrikanisierung“ der Menschenrechte Tonndorf, Menschenrechte in Afrika, Konzeption, Verletzung und Rechtsschutz im Rahmen der OAU, Freiburg 1997, S. 100 ff.

Siehe Art. 18 Abs. 3 AfrC.

Hierzu im Einzelnen Wittinger, VRÜ 33 (2000), 470 ff. (481ff.).

Abschnitt 5 u. 11 Präambel der AfrC sowie Art. 17 Abs. 3 AfrC, Art. 18 Abs. Abs. 2 AfrC, Art. 29 Abs. 7 AfrC.

Zur Bedeutung der Tradition in der AfrC insgesamt Matringe, Tradition et modernité dans la Charte africaine des droits de l'homme et des peuples, Brüssel 1996.

Hierzu näher Wittinger, Familien und Frauen im regionalen Menschenrechtsschutz, Baden-Baden 1999, S. 211-224 und $252 \mathrm{ff}$.

Vgl. Punkt 5 der Mauritius-Erklärung. 
klang stehen. Damit wird gleichzeitig eingeräumt, dass es auch negative afrikanische Traditionen gibt, die diesen Werten widersprechen. Die oben aufgeführten Beispiele afrikanischer Traditionen fallen unter diese letztere Kategorie negativer, menschenrechtsverletzender Praktiken, die nicht in Menschenrechtsdokumenten geschützt werden können. Unklare Formulierungen in der Afrikanischen Menschenrechtscharta können damit von der Afrikanischen Menschenrechtskommission ${ }^{56}$ und dem zukünftigen Afrikanischen Menschenrechtsgerichtshof mit Hilfe der Mauritius-Erklärung entsprechend ausgelegt und korrigiert werden. ${ }^{57}$ Auch an späterer Stelle wird in der Mauritius-Erklärung ausdrücklich betont, dass die Rechte von Frauen und Kindern garantiert werden müssen. ${ }^{58}$ Die Ausarbeitung eines Zusatzprotokolls zur Afrikanischen Charta über die Rechte von Frauen wird begrüßt und darauf hingewiesen, dass die Staaten auf die Abschaffung kultureller Praktiken, die Frauen - so wörtlich - „entmenschlichen und erniedrigen“ ${ }^{59}$, hinarbeiten müssen. Auch hierin ist eine deutliche Absage insbesondere an den Brauch der Geschlechtsverstümmelung an Mädchen und Frauen zu sehen. Ferner soll die Praktik, Kinder als Soldaten einzusetzen, gestoppt werden, ebenso sollen Kinderarbeit, die sexuelle Ausbeutung von Kindern und Kinderhandel verhindert werden. ${ }^{60}$ Auch der Schutz von Flüchtlingskindern wird angemahnt - eine Reaktion auf die gestiegene Anzahl der Flüchtlinge und Waisen infolge der ethnischen Auseinandersetzungen. Schließlich wird festgestellt, dass die Rechte von HIV-Infizierten, vorrangig wieder Frauen und Kinder, nicht in allen afrikanischen Staaten ausreichend respektiert werden. ${ }^{61}$

\section{b) Gründe für die Menschenrechtsverletzungen in Afrika}

Auf die Feststellung der Bedeutung der Menschenrechte in Afrika und das Aufzeigen von Missständen folgt eine Auflistung der Ursachen von Menschenrechtsverletzungen in den afrikanischen Staaten: u.a. nennt die Mauritius-Erklärung den Rassismus, die Umsiedlung von Völkern, das Schuldenproblem, Gewaltmonopole, fehlende Verantwortlichkeit bei der Verwaltung öffentlicher Angelegenheiten, mangelnde richterliche Unabhängigkeit, - wieder - die schädlichen traditionellen Praktiken, eine unzureichende Pressefreiheit, verfassungswidrige Regierungseinsetzungen und die Ausnutzung ethnischer Zugehörigkeit. ${ }^{62}$

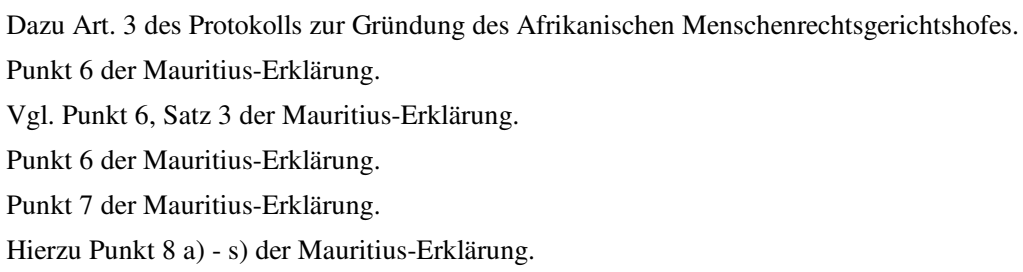


Nach dieser - recht offenen und schonungslosen - Bestandsaufnahme gilt der Blick den Möglichkeiten, die Menschenrechtslage zu verbessern. Hierzu gehört der zweite Schwerpunkt der_Mauritius-Erklärung:

c) Die Bedeutung der Afrikanischen Menschenrechtscharta und die Maßnahmen zur Stärkung der Afrikanischen Menschenrechtskommission

Im Hinblick auf die Afrikanische Menschenrechtscharta ist zunächst festzuhalten, dass die afrikanischen Staaten in der Mauritius-Erklärung aufgefordert werden, die Charta innerstaatlich umzusetzen. ${ }^{63}$ Insoweit bleibt die Erklärung allerdings hinter der Afrikanischen Menschenrechtscharta selbst zurück, denn in der Charta werden die Staaten konkret zur Ergreifung von gesetzgeberischen und sonstigen Maßnahmen angehalten, um die dortigen verbrieften Rechte zu verwirklichen. Weiter widmet sich die Mauritius-Erklärung dem Staatenberichtssystem nach Art. 62 AfrC, wonach die Staaten verpflichtet sind, der Kommission alle zwei Jahre einen Bericht zur Kontrolle der Umsetzung der Staatenverpflichtungen vorzulegen. ${ }^{64}$ In der Mauritius-Erklärung wird angemahnt, dass die Staaten die Erfüllung ihrer Berichtspflichten gewährleisten sollen. Damit wird auf die Schwächen bei der Berichtsmoral der Mitgliedstaaten ${ }^{65}$ bzw. auf vorgelegte, wenig aussagekräftige und unzureichende Berichte ${ }^{66}$ reagiert.

Bezüglich der Afrikanischen Menschenrechtskommission nimmt die Mauritius-Erklärung mehrfach Stellung. Zum einen wird an die Afrikanische Menschenrechtskommission appelliert, Strategien zu entwickeln, um die afrikanische Bevölkerung über Menschenrechte $\mathrm{zu}$ informieren und zu Menschenrechten zu erziehen; konkret wird insoweit vorgeschlagen, in die Stundenpläne der Schulen entsprechende Veranstaltungen aufzunehmen. ${ }^{67}$ Hiermit wird dem Umstand Rechnung getragen, dass die Existenz der Afrikanischen Menschenrechtscharta in Afrika noch immer nicht allzu bekannt ist. ${ }^{68}$ Zum anderen werden auch bei der Afrikanischen Menschenrechtskommission die Schwierigkeiten in der Praxis ihrer Arbeit erkannt: Es wird eingeräumt, dass die Strukturen und das Arbeiten der Kommission gestärkt werden müssen und appelliert, die Kommission mit den nötigen finanziellen und personellen Mitteln auszustatten. ${ }^{69}$ Auch hinsichtlich der bisherigen nach der Charta beste-

63

64

65

66

67

68

69

Punkt 14 der Mauritius-Erklärung.

Hierzu Worku, Entwicklungstendenzen des regionalen Menschenrechtsschutzes, Die Afrikanische Charta der Rechte des Menschen und der Völker, Berlin 2000, 120 ff.

Siehe auch Malmström / Oberleitner, Netherlands Quarterly of Human Rights 10 (1996), 92 f.

Dazu Wittinger, Nachweis in Fn. 54, S. 208 ff. (in Bezug auf Art. 18 Abs. 3 AfrC).

Punkt 20 der Mauritius-Erklärung.

Hierzu Benedek, Zeitschrift für ausländisches öffentliches Recht und Völkerrecht 54 (1994), 169.

Punkt 23 der Mauritius-Erklärung. 
henden Pflicht der Kommission, ihren Aktivitätsbericht der OAU-Versammlung der Staatsund Regierungschefs vorzulegen ${ }^{70}$, enthält die Mauritius-Erklärung eine Abweichung. Höflich formuliert, wird vorgeschlagen, dass diese Aufgabe künftig dem OAU-Ministerrat zufallen möge. Insoweit präsentiert die Mauritius-Erklärung allerdings eine sehr fragliche Lösung. Die einzig korrekte Regelung bestünde darin, der Afrikanischen Menschenrechtskommission selbständig und unabhängig von einem politischen Kontrollorgan die Veröffentlichung ihrer Aktivitätsberichte zu ermöglichen. Denn der Ministerrat, bestehend aus den Außenministern oder anderen Ministern der OAU-Staaten, stellt ebenso ein politisches Organ dar wie die Versammlung der Staats- und Regierungschefs. ${ }^{71}$ Warum die MauritiusErklärung hier die Verlagerung auf den Ministerrat propagiert, darüber kann nur spekuliert werden. Möglicherweise wird intern eine Ausweitung von Organkompetenzen gegenüber der OAU-Versammlung angestrebt, möglicherweise sind auch Praktikabilitätsgründe verantwortlich, da sich die OAU-Versammlung der Staats- und Regierungschefs nur einmal im Jahr trifft, der Ministerrat aber mehrmals.

\section{d) Das Handlungsprogramm}

Der dritte Schwerpunkt betrifft den Aktionsplan, also die Maßnahmen, die ergriffen werden sollen, um das Ziel der Mauritius-Erklärung, die Stärkung der Menschenrechte, zu realisieren. Der Plan wendet sich an drei Adressaten:

Zuvörderst an die Staaten. Diese werden z.B. dazu angehalten, eine freie Presse zu ermöglichen und die NGOs konstruktiv bei ihrer Arbeit zu unterstützen. Im Einzelnen bleibt die Umsetzungsart überwiegend den Staaten überlassen und werden die geeigneten Maßnahmen nicht näher definiert. ${ }^{72}$ Konkret werden die OAU-Staaten allerdings dazu aufgefordert, die Menschenrechtsdokumente der OAU und internationale Instrumente zu ratifizieren: u.a. das Zusatzprotokoll über die Schaffung des Afrikanischen Menschenrechtsgerichtshofs und das Statut des Internationalen Strafgerichthofes. ${ }^{73}$ In bezug auf letzteren wird der Umgang mit ehemaligen Diktatoren Afrikas auch einen entscheidenden Anteil zur Befriedung und Demokratisierung in den afrikanischen Staaten einnehmen; innerstaatlich haben die afrikanische Gerichte bereits begonnen, ehemalige Staatschefs zu verfolgen und Vermögen einzufrieren, aber auch andere afrikanische Organe, z.B. die südafrikanische Wahrheitskommission, oder internationale Instanzen, wie das Ruandatribunal oder auch ausländische Gerichte, wie die belgischen, haben hier ihren Anteil geleistet; Voraussetzung ist jedoch, wie bereits beim künftigen Afrikanischen Menschenrechtsgerichtshof, dass die Staaten

70

71

72

73

Art. 54 AfrC.

Zum OAU-System Kunig, Das völkerrechtliche Nichteinmischungsprinzip, Baden-Baden 1981, $81 \mathrm{ff}$.

Vgl. z.B.: Punkt 6, 9 und 11 der Mauritius-Erklärung.

Dazu Punkt 13 a) - m) der Mauritius-Erklärung. 
überhaupt willens sind, sich einer internationalen Gerichtsbarkeit zu unterwerfen. Nicht zuletzt empfiehlt die Mauritius-Erklärung den Staaten, nationale plans of action zur Förderung und zum Schutz der Menschenrechte anzunehmen. ${ }^{74}$

Zum zweiten wendet sich der Aktionsplan an die OAU. Es wird gefordert, dass der Schutz der Menschenrechte in alle Aktivitäten der OAU integriert werden und die Ministerratskonferenz über Menschenrechte zu einer ständigen Einrichtung werden soll. ${ }^{75}$ Dem OAU Generalsekretär wird - ebenso wie der Afrikanischen Kommission - aufgegeben, Strategien zu erarbeiten, um die Bevölkerung über Menschenrechte aufzuklären. ${ }^{76}$ Zusammen mit den Staaten soll die OAU ferner Lösungen für das Flüchtlingsproblem suchen. ${ }^{77}$

Zum dritten wendet sich die Mauritius-Erklärung auch an internationale Organisationen und an die internationale Gemeinschaft. Diese werden daran erinnert, zur Entschuldung der afrikanischen Staaten beizutragen. ${ }^{78}$ Insofern wird am Ende des Dokuments der Bedeutung, die die Mauritius-Erklärung der Verbesserung der ökonomischen Situation als Basis für den Schutz der Menschenrechte in Afrika zumisst, noch einmal Rechnung getragen.

\section{Fazit}

Ein Fazit muss zwischen der politischen und der rechtlichen Wirkung der MauritiusErklärung unterscheiden.

Politisch gesehen, ist die Mauritius-Erklärung ein klares Bekenntnis dazu, dass der Schutz der Menschenrechte in Afrika nicht mit dem bisher Erreichten - auch nicht mit dem Protokoll zur Gründung des Afrikanischen Menschenrechtsgerichtshofes - als abgeschlossen betrachtet wird. Die Mauritius-Erklärung macht deutlich, dass den Menschenrechten innerhalb der OAU zukünftig eine Priorität eingeräumt werden muss; dies wird im übrigen auch für die Afrikanische Union gelten müssen, die Ende Mai 2001 gegründet wurde und in die sich die OAU umwandeln will. Außerdem ist die Mauritius-Erklärung eine Bilanz der tatsächlichen Menschenrechtslage in Afrika und benennt die Missstände und ihre Ursachen. Dies stellt den ersten Schritt und die Voraussetzung für die Bekämpfung von Menschenrechtsverletzungen dar. 
Aus rechtlicher Sicht ist im Hinblick auf die Konsequenzen der Mauritius-Erklärung festzuhalten, dass sie keinen Vertragscharakter hat und nicht bindend ist. Sie ist die Erklärung eines Organs der OAU, des Ministerrats in Form der Ersten Ministerkonferenz über Menschenrechte, der auch aufgrund seiner Stellung im OAU-Gefüge, nicht befugt ist, den Mitgliedstaaten Verpflichtungen aufzuerlegen. Andererseits heißt dies nicht, dass die Mauritius-Erklärung ohne jede Wirkung sein muss. Einmal können die OAU-Staaten durchaus die Erklärung innerstaatlich, wie gefordert, durch entsprechende Handlungsprogramme rechtlich fassbar umsetzen. Zum zweiten bringt die Mauritius-Erklärung einen Konsens der OAU-Staaten zum Ausdruck und liefert damit Argumente für die Auslegung der Afrikanischen Menschenrechtscharta. ${ }^{79}$ Im Bereich des Begriffes der „Traditionen“, negativer kultureller Praktiken und der Rechte von Frauen und auch Kindern schafft die MauritiusErklärung Klarheit, indem sie sich lediglich zu „positiven“ afrikanischen Traditionen bekennt und ist damit - obwohl ,soft law“ - ein geeignetes Interpretationsmittel, um unklare Formulierungen in der Afrikanischen Menschenrechtscharta entsprechend auszulegen.

\section{Gesamtbewertung}

Als Gesamtergebnis ist damit festzuhalten: die Schaffung des Gerichtshofes bedeutet einen wichtigen Schritt für das Individuum und für NGOs, die Sanktionierung von Menschenrechtsverletzungen im Einzelfall zu erreichen; daneben wird allerdings die Arbeit der Afrikanischen Menschenrechtskommission nicht hinfällig, da sie über den individuellen Fall hinaus, Menschenrechtsverletzungen untersuchen kann und sie Informations- und Aufklärungsfunktionen erfüllt. Die Mauritius-Erklärung will die Ursachen von Menschenrechtsverletzungen bekämpfen und die Gesamtsituation der Menschenrechte in Afrika durch entsprechende Maßnahmen vor allem seitens der Staaten verbessern. Zusammen betrachtet, ergänzen sich der zukünftige Afrikanische Menschenrechtsgerichtshof und die MauritiusErklärung. Beide können auf ihre Weise zur Stärkung der Menschenrechte in Afrika beitragen. 